\title{
A novel ensemble bivariate statistical evidential belief function with knowledge-based analytical hierarchy process and multivariate statistical logistic regression for landslide susceptibility mapping
}

\begin{abstract}
This study compares the landslide susceptibility maps from four application models, namely, (1) the bivariate model of the Dempster-Shafer based evidential belief function (EBF); (2) integration of the EBF in the knowledge-based analytical hierarchy process (AHP) as a pairwise comparison model processed by using all available causative factors; (3) integration of the EBF in the knowledge-based AHP as a pairwise comparison model by using high nominated causative factor weights only; and (4) integrated EBF in the logistic regression (LR) as a multivariate model by using nominated causative factor weights only. These models were tested in Pohang and Gyeongju Cities (South Korea) by using the geographic information system GIS platform. In the first step, a landslide inventory map consisting of 296 landslide locations were prepared from various data sources. Then, a total of 15 landslide causative factors (slope angle, slope aspect, curvature, surface roughness, altitude, distance from drainages, stream power index, topographic wetness index, wood age, wood diameter, wood type, forest density, soil thickness, soil texture, and soil drainage) were extracted from the database and then converted into a raster. Final susceptibility maps exhibit close results from the two models. Models 1 and 3 predicted $82.3 \%$ and $80 \%$ of testing data during the analysis, respectively. Thus, Models 1 and 3 show better performance than LR. These resultant maps can be used to extend the capability of bivariate statistical based model, by finding the relationship between each single conditioning factor and landslide locations, moreover, the proposed ensemble model can be used to show the inter-relationships importance between each conditioning factors, without the need to refer to the multivariate statistic. The research outcome may provide powerful tools for natural hazard assessment and land use planning.
\end{abstract}

Keyword: Ensemble model; Landslide susceptibility; Remote sensing; Geographic information system (GIS); South Korea 